\title{
Disk Stability and Turbulence Generation: Effects of the Stellar Component
}

\author{
Woong-Tae Kim \\ Department of Physics and Astronomy, FPRD, Seoul National University, Seoul 151-742, \\ Republic of Korea \\ email: wkim@astro.snu.ac.kr
}

\begin{abstract}
Galactic disks consist of both stars and gas. The stars gravitationally influence the gas either in disks at large or within spiral arms, leading to the formation of giant clouds and turbulence driving in the gas. In featureless disks as in flocculent galaxies, swing amplification operating in a combined star-gas disk is efficient to form bound condensations and feed a significant level of random gas motions. This occurs when the gaseous Toomre parameter is less than 1.4 for the stellar parameters similar to the solar neighbourhood conditions. In disks with spiral features, on the other hand, spiral-arm spurs and associated giant clouds develop as a consequence of magneto-Jeans instability in which magnetic tension counterbalances the stabilizing Coriolis force. Spiral shocks are inherently unstable when the vertical dimension is taken into account, exhibiting flapping motions of the shock front. This naturally converts the kinetic energy in galaxy rotation into random kinetic energy of the gas. The resulting turbulent motions are supersonic and persist despite strong shock dissipation. Thermal instability occurring in gas flows across spiral arms prompts phases transitions that produce a significant fraction of thermally-unstable, intermediate-temperature gas in the postshock expansion zones.
\end{abstract}

Keywords. galaxies: ISM, instabilities, ISM: kinematics and dynamics, ISM: magnetic fields, method: numerical, stars: formation

\section{Introduction}

Understanding the physical processes that regulate star formation in disk galaxies is of paramount importance for galaxy formation and evolution, which is one of the main themes of this symposium. The fact that most star formation in the Milky Way and external disk galaxies takes place in cold, giant molecular clouds (GMCs) (e.g., Williams, Blitz, \& McKee 2000) implies that the compression of diffuse gas (or small clouds) into GMCs is the first step in initiating galactic star formation. The fact that the largest GMCs, including atomic envelopes, typically have mass $\sim 10^{7} M_{\odot}$ and spacing $\sim 1 \mathrm{kpc}$ (e.g., Elmegreen 1995a and references therein), similar to the characteristic Jeans mass and wavelength in galactic gaseous disks at large, strongly supports the idea that GMCs originate from large-scale gravitational instabilities (e.g., Elmegreen \& Elmegreen 1983). Although Parker instability has also been proposed as a candidate for GMC formation (Blitz \& Shu 1980), it is stabilized by magnetic tension at the non-linear stage and is thus unable to produce high-density structures like GMCs (e.g., Santillán et al. 2000; Kim \& Ostriker 2006).

Turbulence appears to dominate the internal dynamics responsible for fragmentation, compression/dispersal, and hierarchical structures of GMCs and plays an important role in controlling star formation within GMCs (e.g., Elmegreen 2007; McKee \& Ostriker 2007). Various mechanisms have been proposed for interstellar turbulence, including ones based on stellar energies such as expansion of H II regions, outflows from young stellar 
objects, supernova explosions, etc., and ones based on non-stellar sources such as magnetorotational instability, gravitational instability, and galactic spiral shocks, etc. (see Mac Low \& Klessen 2004; Elmegreen \& Scalo 2004; Ostriker 2006 for recent reviews). Stellar sources have often been favoured since supernovae at the Galactic rate are estimated to produce the observed level of the ISM turbulence. However, radio observations of external galaxies indicate that turbulent $\mathrm{H}$ I velocity dispersions in non-star-forming outer disks are comparable to those in star-forming inner disks (van Zee \& Bryant 1999) and they are not correlated with spiral arms, as well (Petric \& Rupen 2007). These invoke the possibility of turbulence driving by non-stellar sources, many of which simply transport kinetic energy in disk rotation into random gas motions.

In this article we review recent numerical studies on GMC formation and turbulence generation via gravitational instability and other dynamical processes in disk galaxies, concentrating on the role played by stellar gravity. In addition to galactic differential rotation, thermal/turbulent pressure, self-gravity, and magnetic fields, gas in disk galaxies is also influenced by gravity from a dynamically-active stellar disk or by stellar spiral potential perturbations. For more detailed, quantitative results, the reader is refereed to Kim et al. (2006, 2008) and Kim \& Ostriker $(2006,2007)$.

\section{Gravitational Instabilities in Featureless Disks}

\subsection{Axisymmetric Instability}

Disk galaxies contain stellar and gaseous disks with finite vertical thickness. Despite large velocity dispersions, the large mass fraction $(\sim 75 \%-90 \%)$ of the stellar disk makes its contribution to the growth of self-gravitating modes in the combined system almost comparable to that of the gaseous part. Most previous works on the effects of the live stellar component approximated stars as an isothermal "fluid" (e.g., Jog \& Solomon 1984, Elmegreen 1995b, Jog 1996). Taking a true kinetic treatment of the stellar component, Rafikov (2001) derived the dispersion relation for axisymmetric modes in razor-thin, twocomponent disks. Taking allowance for the dilution of self-gravity due to finite vertical thickness, the axisymmetric dispersion relation becomes

$$
\frac{2 \pi G k \Sigma_{g}}{\left(\kappa^{2}+k^{2} c_{g}^{2}-\omega^{2}\right)\left(1+k H_{g}\right)}+\frac{2 \pi G k \Sigma_{s} \mathcal{F}\left(\omega / \kappa, k^{2} \sigma_{R}^{2} / \kappa^{2}\right)}{\left(\kappa^{2}-\omega^{2}\right)\left(1+k H_{s}\right)}=1,
$$

where $\omega$ and $k$ denote the frequency and wavenumber of perturbations, respectively, $H_{g}$ and $H_{s}$ are the vertical scale heights of the gaseous and stellar disks, respectively, and $\mathcal{F}$ is the stellar reduction factor (Kim \& Ostriker 2007). Other symbols have their usual meanings. For razor-thin disks $\left(H_{g}=H_{s}=0\right)$, equation (2.1) yields the sufficient conditions $Q_{g} \equiv \kappa c_{g} / \pi\left(G \Sigma_{g}\right)>1$ for gas-only disks and $Q_{s} \equiv \kappa \sigma_{R} /\left(3.36 G \Sigma_{s}\right)>1$ for star-only disks.

Figure 1a plots the marginal stability curves $\left(\omega^{2}=0\right)$ on the $Q_{g}-Q_{s}$ plane for some values of $R \equiv c_{g} / \sigma_{R}$. The results of Rafikov (2001) for razor-thin disks with are plotted as thin lines, while thick lines are for disks with finite thickness with $H_{g}=0.87 c_{g} / \kappa$ and $H_{s}=0.4 \sigma_{R} / \kappa$. Note that a two-component disk marked by a filled circle at $\left(Q_{g}, Q_{s}\right)=$ $(1.4,2.1)$ and $R=0.3$ corresponding to the solar neighborhood conditions, is highly stable to axisymmetric perturbations when finite disk thickness is considered, while it would be marginally stable if treated as razor-thin. Figure 1 b plots the critical $Q_{g}$ values as functions of the gaseous scale height. Clearly, the presence of the stellar component makes the gaseous disk more unstable, increasing $Q_{g, c}$ from unity to 1.27 for razor-thin disks. On the other hand, finite disk thickness decreases this value to $Q_{g, c} \simeq 0.67$ when 

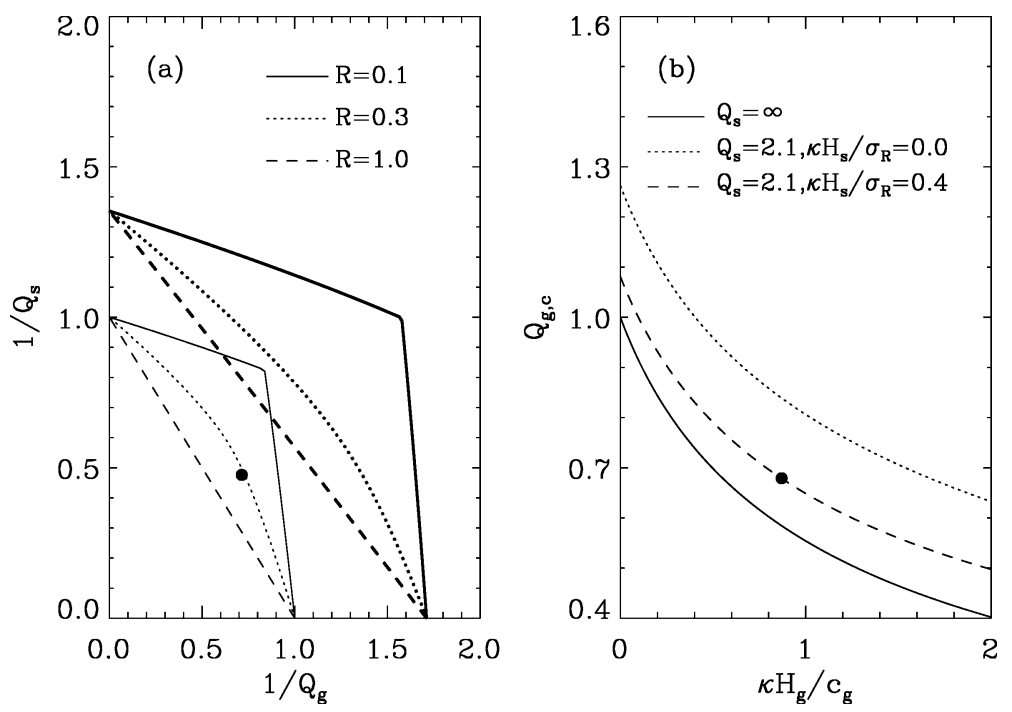

Figure 1. (a) Marginal stability curves for axisymmetric gravitational instability in two-components disks with $R \equiv c_{g} / \sigma_{R}$; the region below each curve represents stable configurations. Thin lines correspond to razor-thin disks, while thick lines are for disks with $H_{g}=0.87 c_{g} / \kappa$ and $H_{s}=0.4 \sigma_{R} / \kappa$. (b) The critical $Q_{g}$ values for gas-only disks (solid line), for combined disks with a razor-thin, stellar component (dotted line), and combined disks with a thick, stellar component (dashed line). In both panels, the dots represent the solar neighborhood conditions.

$H_{g}=0.87 c_{g} / \kappa$ and $H_{s}=0.4 \sigma_{R} / \kappa$, indicating that the stabilizing effect of disk thickness overwhelms the destabilizing effect of stellar gravity. Given that $Q_{g, c} \simeq 0.67$ is well below observed critical values at $Q_{g} \sim 1.4$ (Martin \& Kennicutt 2001), star formation thresholds are unlikely due to axisymmetric gravitational instability in two-component disks.

\subsection{Swing Amplification}

Even if disks are stable to axisymmetric perturbations, nonaxisymmetric modes in shearing disks are allowed to grow as they swing from leading to trailing configurations. This occurs due to conspiracy among rotational shear, self-gravity, and epicycle shaking (Toomre 1981). While the amplitude of density growth in swing is a continuous function of $Q_{g}$ in the linear theory, nonlinear simulations show that disks exhibit $Q_{g}$ threshold behavior such that swing-amplified disturbances in disks with $Q_{g}$ small enough become nonlinear and subsequently gravitationally unstable. Kim \& Ostriker (2001) found that the critical values for nonlinear threshold are $Q_{g, c} \sim 1.2-1.4$ for razor-thin, gas-only disks. By solving the orbits of stars using a particle-mesh method (instead of treating them as a fluid) and evolving the gaseous disk based on a hydrodynamic method, Kim \& Ostriker (2007) found $Q_{g, c} \sim 1.4$ for thick, two-component disks with the stellar parameters similar to the solar neighborhood values. Similar critical values were obtained by Li et al. 2006 for global galaxy models. Disks with $Q_{g}$ less than the critical value develop high-density filaments that eventually undergo fragmentation and produce gravitationally bound condensations of mass $6 \times 10^{7} M_{\odot}$ each.

In addition to forming bound condensations, swing amplification in two-component disks can also be vigorous enough to tap rotational and gravitational energy to feed random motions in the gaseous component. When $Q_{g} \simeq Q_{g, c}$, the gaseous velocity dispersions induced in two-component disks amount to $\sim 0.6 c_{g}$, which is $\sim 3-4$ times larger than the values obtained from gas-only disks (Kim \& Ostriker 2007). This level of 
turbulence driven by swing amplification in marginally-stable disks is comparable to that by magnetorotational instability in non-self-gravitating, gas-only disks (Kim et al. 2003). The turbulence driven by swing is anisotropic. The ratio of total power in the shearing to compressive parts is about 1.5, which is $\sim 3-7$ times lower than found in hypersonic turbulence. This indicates that the turbulence in two-component models is subsonic and that the pumping by self-gravity is preferentially via compressional motions.

\section{Galactic Spiral Shocks}

\subsection{Magneto-Jeans Instability}

The stellar component has a dramatic effect on the evolution of the gaseous component through spiral density waves. Being dynamically much more responsive, the gas passing through the gravitational potential wells of stellar spiral arms is strongly compressed and shocked, producing narrow dust lanes with strong magnetic fields. In addition, the conservation law of angular momentum requires that shear be reversed inside spiral arms, making the overall shear experienced by gas traversing the arm regions very small. These imply that spiral arms are prone to magneto-Jeans instability (Lynden-Bell 1966; Elmegreen 1994; Kim \& Ostriker 2002) in which magnetic tension forces negate the stabilizing effects of galaxy rotation. When finite disk thickness is considered, the most unstable disturbances have wavelengths about 10 times the Jeans length at the arm peaks (Kim \& Ostriker 2006). They first grow in the direction perpendicular to the arms before trailing away in the interarm regions, similar to dust filaments or spurs (feathers) found in recent Spitzer and HST images of M51. In the nonlinear stage, spurs experience fragmentation to produce bound clumps of mass $\sim 10^{7} M_{\odot}$ each, similar to the largest observed GMCs, which may evolve to H II regions in both arm and interarm regions.

\subsection{Turbulence Driving}

Spiral shocks act as one of the important mechanisms that transform a fraction of kinetic energy in galaxy rotation into gaseous turbulence. One-dimensional spiral shocks with fluid quantities varying only along the direction perpendicular to the shocks are known to readily stationary, indicating that they represent stable equilibria (Roberts 1969 Woodward 1975; Kim \& Ostriker 2002). When the vertical degrees of freedom are allowed, however, spiral shocks never achieve a steady state, moving back and forth relative to the mean positions (Kim \& Ostriker 2006). These shock flapping motions arise mainly because the vertical oscillation period of the gas is incommensurable with the arm-toarm crossing time, so that the gas streamlines are not closed. The shock flapping is able to feed random gas motions on the scale of disk scale height that persist despite shock dissipation. The induced velocity dispersions amount to $\sim 7-10 \mathrm{~km} \mathrm{~s}^{-1}$ in the plane and $\sim 3-4 \mathrm{~km} \mathrm{~s}^{-1}$ in the vertical direction, and are larger inside spiral arms than in interarm regions by about a factor of 2 (Kim \& Ostriker 2006).

\subsection{Effect of Thermal Instability}

One of the main characteristics of galactic spiral shocks is that strongly compressed gas at the shock front becomes less dense as it expands in the postshock regions. This implies that the gas may undergo thermal phase transitions when the shocked gas is subject to thermal instability. Recently, Kim et al. (2008) explored nonlinear evolution of galactic gas flows across spiral arms that are explicitly exposed to interstellar heating, cooling and thermal conduction. Figure 2 plots an exemplary density profile, when a quasi-steady state is reached, of a spiral shock in a one-dimensional model with the initial number density $n_{0}=2 \mathrm{~cm}^{-3}$ and the arm strength $F=5 \%$. In the interarm regions $\left(x / L_{x}<0\right.$ 


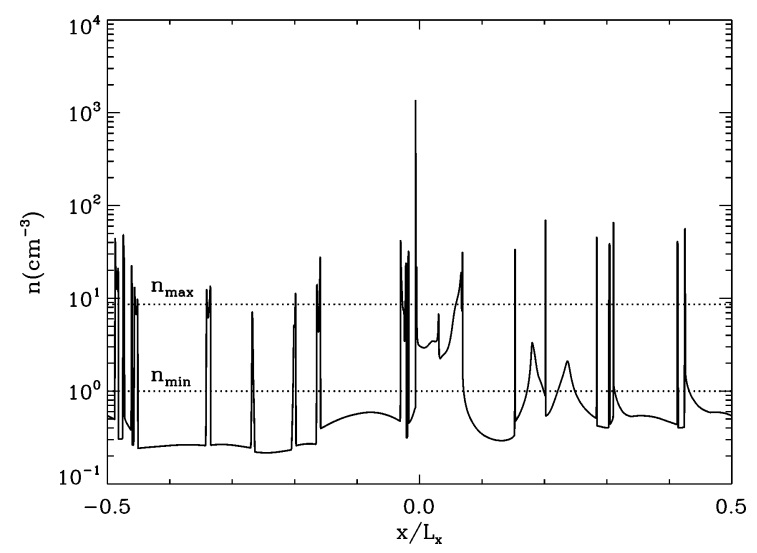

Figure 2. Density profile of a quasi-stationary galactic spiral shock with thermal instability in a model with the initial number density of $n_{0}=2 \mathrm{~cm}^{-3}$. Dotted lines marked by $n_{\max }=8.6 \mathrm{~cm}^{-3}$ and $n_{\mathrm{min}}=1.0 \mathrm{~cm}^{-3}$ divide the cold $\left(n>n_{\max }\right)$, intermediate-temperature $\left(n_{\max }>n>n_{\min }\right)$, and warm $\left(n<n_{\mathrm{min}}\right)$ phases.

or $x / L_{x}>0.2$ ) cold clouds with $n>n_{\max }=8.6 \mathrm{~cm}^{-3}$ are roughly in pressure balance with the surrounding warm intercloud medium with $n<n_{\min }=1 \mathrm{~cm}^{-3}$.

The warm/cold gas in the interarm regions is shocked and immediately turns to denser cold gas in the arm region $\left(0<x / L_{x}<0.03\right)$. Due to the post-shock expansion, this subsequently enters the unstable transition zone $\left(0.03<x / L_{x}<0.2\right)$ and undergoes thermal instability to evolve back to the warm/cold interarm phases. For the model shown in Figure 2, the gas stays at the arm, transition, and interarm zones approximately for $14 \%, 22 \%$, and $64 \%$ of the arm-to-arm crossing time, respectively. The gas at intermediate temperature (with $n_{\min }<n<n_{\max }$ ) is found to be $\sim 0.25-0.30$ of the total by mass. Of this, about $70 \%$ is found in the transition zone, while the remaining $30 \%$ corresponds to the boundaries of the cold interarm clouds, suggesting that the postshock expanding flows are an important source for the intermediate-temperature gas. Kim et al. (2008) also found that thermal instability in association with one-dimensional spiral shocks can drive random gas motions at $\sim 1.5 \mathrm{~km} \mathrm{~s}^{-1}$ in the interarm and transition zones, which is $\sim 5-7$ times larger than the values from pure thermal instability (e.g., Kritsuk \& Norman 2002; Piontek \& Ostriker 2004). Given that one-dimensional isothermal spiral shocks are stable, these results suggest that flapping motions with thermal instability in multi-dimensional spiral shocks can be even more efficient in driving turbulence in the arm and interarm regions.

\section{Summary}

Recent numerical simulations have shown that for the stellar parameters similar to those in the solar neighborhood, a two-component (stars plus gas) disk without spiral features is subject to gravitational runaway provided the gaseous disk has a Toomre stability parameter less than 1.4. In this case, swing amplification is able to form bound clumps and drive a significant level of turbulent energy in the gas. In disks with spiral arms, the gas is easily compressed to form a spiral shock where magneto-Jeans instability is efficient to produce arm substructures such as spurs and giant clouds. Spiral shocks in two or higher dimensions are inherently unstable to generate random gas motions that remain supersonic even in the presence of shock dissipation. In short, the effect of the stellar component on disk instability and turbulence driving is by no means negligible. 


\section{Acknowledgements}

Much of the material contained in this article is based on the collaborative work with E. Ostriker and C.-G. Kim. The financial support from the organizers of this symposium is gratefully acknowledged. This work has been supported in part by Korea Research Foundation Grant funded by the Korean Government (MOEHRD) (KRF-2007-313-C00328).

\section{References}

Blitz, L. \& Shu, F. H. 1980, ApJ, 238, 148

Elmegreen, B. G. 2007, ApJ, 668, 1064

Elmegreen, B. G. 1995a, in The 7th Guo Shoujing Summer School on Astrophysics: Molecular Clouds and Star Formation, eds. C. Yuan \& Hunhan You (Singapore:World Scientific), 149

Elmegreen, B. G. 1995b, MNRAS, 275, 944

Elmegreen, B. G. \& Elmegreen, D. M. 1983, MNRAS, 203, 31

Elmegreen, B. G. \& Scalo, J. 2004, ARAA, 42, 211

Elmegreen, B. G. 1994, ApJ, 433, 39

Jog, C. J. 1996, MNRAS, 278, 209

Jog, C. J. \& Solomon, P. M. 1984, ApJ, 276, 127

Kim, C.-G., Kim, W.-T., \& Ostriker, E. C. 2006, ApJ, 649, L13

Kim, C.-G., Kim, W.-T., \& Ostriker, E. C. 2008, ApJ, in press; arXiv:0804.0139

Kim, W.-T. \& Ostriker, E. C. 2001, ApJ, 559, 70

Kim, W.-T. \& Ostriker, E. C. 2002, ApJ, 570, 132

Kim, W.-T. \& Ostriker, E. C. 2006, ApJ, 646, 213

Kim, W.-T. \& Ostriker, E. C. 2007, ApJ, 660, 1232

Kim, W.-T. Ostriker, E. C, \& Stone, J. M. 2003, ApJ, 599, 1151

Kritsuk, A. G. \& Norman, M. L. 2002, ApJ, 569, L127

Li, Y., Mac Low, M.-M., \& Klessen, R. S. 2006, ApJ, 626, 823

Lynden-Bell, D. 1966, Observatory, 86, 57

Mac Low, M.-M. \& Klessen, R. S. 2004, Rev. Mod. Phys., 76, 125

Martin, C. L. \& Kennicutt, R. C. 2001, ApJ, 555, 301

McKee, C. F. \& Ostriker, E. C. 2007, ARAA, 45, 567

Ostriker, E. C. 2006, in IAU Symp. 237, Triggered Star Formation in a Turbulent ISM, ed. B. G. Elmegreen \& J. Palous (Cambridge: Cambridge Univ. Press), 70

Petric, A. O. \& Rupen, M. P. 2007, AJ, 134, 1952

Piontek, R. A. \& Ostriker, E. C. 2004, ApJ, 601, 905

Rafikov, R. R. 2001, MNRAS, 323, 445

Roberts, W. W. 1969, ApJ, 158, 123

Santillán, A., Kim, J., Franco, J., Martos, M., Hong, S.S., \& Ryu, D. 2000, ApJ, 545, 353

Toomre, A. 1981, in Structure and Evolution of Normal Galaxies, eds. S. M. Fall \& D. LyndenBell (Cambridge:Cambridge Univ. Press), 111

van Zee, L., \& Bryant, J. 1999, AJ, 118, 2172

Williams, J. P., Blitz, L., \& McKee, C. F. 2000, in Protostars and Planets IV, eds. Mannings, Boss, \& Russell (Tuscon:Univ. of Arizona press), 97

Woodward, P. R. 1975, ApJ, 195, 61 